\title{
Improving the Performance of a NER System by Post-processing and Voting
}

\author{
Asif Ekbal and Sivaji Bandyopadhyay \\ Department of Computer Science and Engineering \\ Jadavpur University, Kolkata, India-700032 \\ asif.ekbal@gamil.com, sivaji_ju@vsnl.com
}

\begin{abstract}
This paper reports about the development of a NER system in Bengali by combining outputs of the classifiers such as Maximum Entropy (ME), Conditional Random Field (CRF) and Support Vector Machine (SVM). The training set consists of approximately $250 \mathrm{~K}$ wordforms and has been manually annotated with the four major named entity (NE) tags such as Person, Location, Organization and Miscellaneous tags. The classifiers make use of the different contextual information of the words along with the variety of features that are helpful in predicting the various NE classes. Lexical context patterns, which are generated from an unlabeled corpus of 1 million wordforms in a semi-automatic way, have been used as the features of the classifiers in order to improve their performance. In addition, we have used the second best tags of the classifiers and applied several heuristics to improve the performance. Finally, the classifiers are combined using a majority voting approach. Experimental results show the effectiveness of the proposed approach with the overall average recall, precision, and f-score values of $90.78 \%, 87.35 \%$, and $89.03 \%$, respectively, which shows an improvement of $11.8 \%$ in f-score over the best performing SVM based baseline system and an improvement of $15.11 \%$ in f-score over the least performing ME based baseline system. The proposed system also outperforms the other existing Bengali NER system.
\end{abstract}

Keywords: Natural Language Processing, Named Entity Recognition, Maximum Entropy, Conditional Random Field, Support Vector Machine, Majority Voting.

\section{Introduction}

Named Entity Recognition (NER) is an important tool in almost all Natural Language Processing (NLP) application areas including machine translation, question answering, information retrieval, information extraction, automatic summarization etc. The current trend in NER is to use the machine-learning approach, which is more attractive in that it is trainable and adoptable and the maintenance of a machine-learning system is much cheaper than that of a rule-based one. The representative machinelearning approaches used in NER are Hidden Markov Model (HMM) (BBN's IdentiFinder [1]), Maximum Entropy (ME) (New York University's MENE [2]) and Conditional Random Fields (CRFs) [3]. Support Vector Machines (SVMs) based 
NER system was proposed by Yamada et al. [4] for Japanese. The process of stacking and voting method for combining strong classifiers like boosting, SVM and TBL, on NER task can be found in [5]. Florian et al. [6] tested different methods for combining the results of four systems and found that robust risk minimization worked best. Munro et al. [7] employed both voting and bagging for combining classifiers. The work reported in this paper differs from that of $\mathrm{Wu}$ et al. [5], Florian et al. [6] and Munro et al. [7] in the sense that here, we have conducted a number of experiments to post-process the outputs of the classifiers with the lexical context patterns, which are generated in a semi-automatic way from an unlabeled corpus of 1 million wordforms, and used several heuristics to improve the performance of each classifier before applying majority voting.

Named Entity (NE) identification and classification in Indian languages in general and in Bengali in particular is difficult and challenging. Unlike English and European languages, Bengali lacks capitalization information, which plays a very important role in identifying NEs. Indian names are more diverse and a lot of these words can be found in the dictionary with specific meanings. Another problem in Indian language NER is the scarcity of NE annotated corpus. A pattern directed shallow parsing approach for NER in Bengali is reported in [8]. A HMM based NER system for Bengali has been reported in [9], where additional contextual information has been considered during emission probabilities and NE suffixes are kept for unknown word handling. More recently, the works in the area of Bengali NER can be found in [10] and [11] with the CRF, and SVM approach, respectively.

Other than Bengali, the works on Hindi can be found in [12] with CRF, [13] with a language independent method, [14] with a hybrid feature set based ME approach and in [15] using MEMM. As part of the IJCNLP-08 NER shared task, various works of NER in Indian languages using various approaches can be found in NERSSEAL ${ }^{1}$.

\section{Named Entity Recognition in Bengali}

Bengali is the seventh popular language in the world, second in India and the national language of Bangladesh. We have used a Bengali news corpus [16], developed from the web-archive of a widely read Bengali newspaper for NER. Out of 34 million wordforms, $150 \mathrm{~K}$ wordforms have been manually annotated with the four NE tags namely, Person, Location, Organization and Miscellaneous. We have also collected the unannotated corpus of around $100 \mathrm{~K}$ wordforms, particularly from the literature, agriculture and scientific domains and manually annotated with the four NE tags. Both the annotations were carried out by a person and verified by an expert. In order to properly denote the boundaries of NEs, these four NE tags are further divided in the form:

B-XXX: Beginning of a multi-word NE, I-XXX: Internal of a multi-word NE, E-XXX: Ending of multi-word NE (e.g., sachin ramesh tendulkar is tagged as sachin /B-PER ramesh/I-PER tendulkar/E-PER). The single-word NE is tagged as PER/ LOC/ORG/MISC. The words that do not denote the NEs are tagged as NNE.

\footnotetext{
${ }^{1}$ http://ltrc.iiit.ac.in/ner-ssea-08/proc/index.html
} 


\section{Approaches to NER for Bengali}

Incorporating diverse features in an HMM-based NE tagger is difficult and complicates the smoothing typically used in such taggers. In contrast, ME, CRF or SVM based method can deal with the diverse and morphologically complex features of the Indian languages.

In this paper, we have used ME, CRF and SVM frameworks in order to identify NEs from a Bengali text and to classify them into Person, Location, Organization and Miscellaneous. A semi-automatic context pattern induction method, second best tag of the classifier and several heuristics have been used in order to improve the performance of each of the classifiers. Finally, we have combined these classifiers with the help of a simple majority voting scheme.

We have used the $\mathrm{C}++$ based ME package (http://homepages.inf.ed. ac.uk/ s0450736/software/maxent/maxent-20061005.tar.bz2)and $\mathrm{C}^{++}$based OpenNLP CRF++ package (http://crfpp.sourceforge.net) for NER. Support Vector Machines (SVMs) have advantages over conventional statistical learning algorithms, such as Decision Tree, HMMs, ME in terms of its high generalization performance independent of dimension of feature vectors and its ability of learning with all combinations of given features without increasing computational complexity by introducing the kernel function. We have used YamCha (http://chasen-org/ taku/software/yamcha) toolkit, an SVM based tool for detecting classes in documents and formulating the NER task as a sequential labeling problem. Here, the pair wise multi-class decision method and second degree polynomial kernel function were used. We have used TinySVM-0.07 (http://cl.aist-nara.ac.jp/ taku-ku/software/TinySVM) classifier that seems to be the best optimized among publicly available SVM toolkits.

\subsection{Named Entity Features}

Experiments have been carried out in order to find out the most suitable features for NER in Bengali. Following are the details of the set of features that have been applied to the NER task:

- Context words: Preceding and following words of a particular word. This is based on the observation that the surrounding words are very effective in the identification of NEs.

- Word suffix and prefix: Word suffix and prefix information are helpful to identify NEs. A fixed length (say, $n$ ) word suffix/prefix of the current and/or the surrounding word(s) can be treated as feature(s). If the length of the corresponding word is less than or equal to $n-1$ then the feature values are not defined and denoted by ND. The feature value is also not defined (ND) if the token itself is a punctuation symbol or contains any special symbol or digit. Another way to use the suffix information is to modify the feature as binary valued. Variable length suffixes of a word can be matched with predefined lists of useful suffixes (e.g., - babu, -da, -di etc. for persons and -land, -pur, -lia etc. for locations). These features are useful to handle the highly inflective Indian languages like Bengali.

- Named Entity Information (dynamic feature): NE tag(s) of the previous word(s). 
- First word (binary valued): Current token is the first word of the sentence or not. First word is generally NE.

- Length of the word (binary valued): Length of the token is less than three or not. This is based on the observation that the short words are rarely NEs.

- Infrequent word (binary valued): A cut off frequency has been chosen in order to consider the infrequent words in the training corpus. Frequently occurring words are rarely NEs.

- Digit features: Several digit features have been considered depending upon the presence and/or the number of digit(s) in a token. These binary valued features are helpful in recognizing miscellaneous NEs such as time, monetary and date expressions, percentages, numerical numbers etc.

- Position of the word (binary valued): Position of the word in a sentence is a good indicator of NEs. Generally, verbs occur at the last position of the sentence. This feature is used to check whether the word is the last word in the sentence.

- Part of Speech (POS) Information: We have used a CRF-based POS tagger [18] that was originally developed with 26 POS tags, defined for the Indian languages. We have considered a coarse-grained POS tagger that has the following tags: Nominal, PREP (Postpositions) and Other. Postpositions are considered as these often appear after the NEs.

- Gazetteer Lists: Gazetteer lists, developed from the Bengali news corpus [11], have been used as the features in each of the classifiers. If the current token is in a particular list, then the corresponding feature is set to 1 for the current and/or the surrounding word(s); otherwise, it is set to 0 . The following is the list of gazetteers along with the number of entries:

(1).Organization clue word (e.g., kong, limited etc): 94, Person prefixes (e.g., sriman, sreemati etc.): 245, Middle names: 1,491, Surnames: 5,288, Common location (e.g., sarani, road etc.): 547, Action verb (e.g., balen, ballen etc.): 241, Function words: 743, Designation words (e.g., neta, sangsad etc.): 947, First names: 72,206, Location names: 7,870, Organization names: 2,225, Month name (English and Bengali calendars): 24, Weekdays (English and Bengali calendars): 14

(2).Common word (521 entries): Most of the Indian languages NEs appear in the dictionary with some other meanings. For example, the word kamol may be the name of a person but also appears in the dictionary with another meaning lotus, the name of a flower; the word dhar may be a verb or also can be the part of a person name. We have manually created a list, containing the words that can be NEs as well as valid dictionary words.

(3).Lexicon (128,000 entries): We have developed a lexicon from the Bengali news corpus in an unsupervised way. The feature 'LEX' has value 0 for those words that appear in the lexicon; otherwise, the value is 1 . This feature has been considered, as the words that appear in the lexicon are rarely NEs.

\section{Semi-automatic Context Pattern Induction}

For English NER, a context pattern induction method through successive learners is reported in [17]. Here, we have developed a semi-automatic context pattern induction 
method in a different way in order to use the contextual information of words as features of the classifiers as well as to post-process the outputs of each of the classifiers for Bengali NER. For this purpose, we have used an unlabeled corpus of $1000 \mathrm{~K}$ wordforms of the Bengali news corpus [11]. We have collected the frequently occurring words from a part of this news corpus and the training set to use as the seeds. There are 123, 87, and 32 entries in the person, location, and organization seed lists, respectively. The unlabeled corpus is tagged with the elements from the seed lists. For example, $<$ Person $>$ sonia gandhi $</$ Person $>$, <Location $>$ kolkata $</$ Location $>$ and $<$ Organization $>$ jadavpur viswavidyalya $<$ Organization $>$.

For each tag $\mathrm{T}$ inserted in the training corpus, the algorithm generates a lexical pattern $p$ using a context window of maximum width 6 (excluding the tagged $\mathrm{NE}$ ) around the left and the right tags, e.g., $\mathrm{p}=\left[l_{-3} l_{-2} l_{-1}<\mathrm{T}>\ldots</ \mathrm{T}>l_{+1} l_{+2} l_{+3}\right]$, where $l_{ \pm i}$ are the context of p. Any of $1_{ \pm i}$ may be a punctuation symbol. In such cases, the width of the lexical patterns will vary. All these patterns, derived from the different tags of the tagged training corpus, are stored in a Pattern Table (or, set P), which has four different fields namely, pattern id (identifies any particular pattern), pattern example (pattern), pattern type (Person/Location/Organization) and relative frequency (indicates the number of times any particular pattern appears in the entire training corpus relative to the total number of patterns generated). This table has 38,986 entries, out of which 21,031 patterns are distinct.

Every pattern $\mathrm{p}$ in the set $\mathrm{P}$ is matched against the same unannotated corpus of 1 million wordforms. In a place, where the context of $\mathrm{p}$ matches, $\mathrm{p}$ predicts the occurrence of the left or right boundary of name. The POS information of the words as well as well as some linguistic rules is used to detect the other boundary of the NE. In order to do this, the corpus is tagged with the CRF-based POS tagger [18] and then a regular expression Nominal* (NE is a sequence of nominals) has been used. Each new NE (not appearing in the seed lists) so obtained in the unlabeled corpus is manually checked for correctness. The corpus is further tagged with these newly acquired NEs to identify further lexical patterns. The bootstrapping is applied on the training corpus until no new patterns can be generated. The patterns are added to the pattern set $\mathrm{P}$ with the type and relative frequency fields set properly, if they are not already in the pattern set $\mathrm{P}$ with the same type. Any particular pattern in the set of potential patterns $\mathrm{P}$ may occur many times but with different type and with equal or different relative frequency values. For each pattern of the set $\mathrm{P}$, the relative frequencies of its occurrences as person, location and organization names are calculated. For the candidate patterns acquisition, a particular threshold value of relative frequency is chosen. If the relative frequency for a particular pattern (along with the type) is less than this threshold value then this pattern (only for that type) is discarded otherwise, it is retained in the Pattern Table (or, set P). An additional 1,078 number of new patterns were generated through bootstrapping.

To extract the candidate patterns from the set of patterns of the Pattern Table, the $\mathrm{NE}$ annotated corpus of $250 \mathrm{~K}$ wordforms have been used as the gold standard development data. Each pattern $\mathrm{p}$ of the set $\mathrm{P}$ is matched against the development set. The identified NE is checked for the correctness against the gold standard tag(s) of that entity/or the sequence of entities. A pattern is penalized if it extracts entities, which belong to the other classes. We consider the extracted entity of a particular class (type) to be an instance of positive example if it is identified by a pattern of that type. 
The extracted entity is counted as an instance of the negative example if it is extracted by pattern of other type or it is not at all a named entity. Let positive (p) and negative (p) be respectively the number of distinct positive and negative examples extracted by a pattern $\mathrm{p}$. Now, we calculate the following function for each pattern $\mathrm{p}$ :

$\operatorname{precision}(\mathrm{p})=\operatorname{positive}(\mathrm{p}) /[\operatorname{positive}(\mathrm{p})+\operatorname{negative}(\mathrm{p})]$

We discard all the patterns $\mathrm{p}$ if the values of precision ( $\mathrm{p}$ ) are below a predetermined threshold value. The remaining patterns are called the high precision patterns and can be very effective for NE identification. We also discard the patterns whose total positive example (distinct) extraction is less than a certain threshold value. The remaining patterns in the set $\mathrm{P}$ form the set of final patterns to be applied during evaluation and this resultant set is denoted by Accept Pattern. There are 12,633 entries in this set.

\section{Evaluation Results}

A set of $250 \mathrm{~K}$ wordforms, collected from the news corpus, has been manually annotated with the four NE tags with the help of Sanchay Editor ${ }^{2}$, a text editor for the Indian languages. Out of $250 \mathrm{~K}$ wordforms, $150 \mathrm{~K}$ wordforms were collected from the news corpus [16] and the remaining $100 \mathrm{~K}$ wordforms have been collected from the agriculture, literature and scientific domains. Around $30 \mathrm{~K} \mathrm{NE}$ tagged corpus is selected as the development set and the rest $220 \mathrm{~K}$ wordforms are used as the training set of each of the classifiers.

A number of experiments have been conducted taking the different combinations of the available words, context, orthographic word level features and gazetteers to identify the best-suited set of features in the ME, CRF and SVM frameworks for NER in Bengali. These are defined as the baseline models.

Evaluation results of the development set are presented in Table 1 in terms of Recall (R), Precision (P) and F-Score (FS) that are defined as below:

Recall $=$ No. of NEs retrieved correctly/Relevant NEs in the gold standard data; Precision $=$ No. of NEs retrieved correctly $/$ No. of NEs identified by the system; $\mathrm{F}-\mathrm{Score}=(2 *$ Recall $*$ Precision $) /($ Recall + Precision $)$.

Table 1. Results of the development set for the baseline models

\begin{tabular}{|l|l|l|l|}
\hline Model & Recall (in \%) & Precision (in \%) & F-Score (in \%) \\
\hline ME & 78.48 & 73.40 & 74.32 \\
\hline CRF & 82.29 & 76.42 & 77.71 \\
\hline SVM & 82.32 & 77.49 & 78.30 \\
\hline
\end{tabular}

Results have demonstrated that the ME based baseline system performs best (f-score $=74.32 \%$ ) for the context window of size three (i.e., previous, current and next word), NE information of the previous word, POS information of the current word, prefixes and suffixes upto three characters of the current word along with the gazetteers and other features. The baseline CRF model has shown best performance

\footnotetext{
${ }^{2}$ Sourceforge.net/project/nlp-sanchay
} 
(f-score $=77.71 \%$ ) for the context window of size five, POS information of the current and previous words along with the other set of features like ME. The SVM based baseline system has performed best among the three models and has demonstrated the f-score value of $78.3 \%$ for the context window of size six, NE information of the previous two words, POS information of the current, previous and the next words along with the other set of features as like ME and CRF. During all the experiments, we have observed that word context, prefixes, suffixes, POS information, dynamic $\mathrm{NE} \operatorname{tag}(\mathrm{s})$ and digit features are the most effective features for NER. Other features are not so effective but helpful in increasing the overall performance of the NER system.

\subsection{Use of Context Patterns as Features}

The Accept Pattern set (discussed in section 4) is used to prepare three lists of the frequently occurring words in the left and/or the right contexts of person, location and organization names. These words are used as the trigger words and are very useful to identify the NEs. A feature 'ContextInformation' is set appropriately if the window [3,3 ] (three words spanning to left and right) of the current word contains any trigger word of a particular NE type. The use of this feature along with the previously dis-

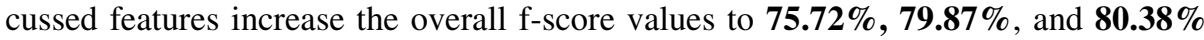
in the baseline ME, CRF, and SVM based systems, respectively. Evaluation results are presented in Table 2.

\subsection{Second Best Tag and Heuristics}

If the best tag given by any of the classifiers is "NNE" (other than NE) and the confidence of the second best tag is greater than a particular threshold value then the second best tag is considered as the correct tag. We conducted a number of experiments to identify the threshold value for each of the classifiers. Finally, the threshold value is set to $0.19 \%$ for ME and $0.17 \%$ for CRF and SVM. Evaluation results of the system are presented in Table 2. Results show an improvement in the recall values by $9.05 \%$, $9.31 \%$, and $9.69 \%$ in the ME, CRF, and SVM, respectively. But, the choice of second tag decreases the precision values by $1.42 \%, 2.44 \%$, and $2.65 \%$ in the ME, CRF, and $\mathrm{SVM}$, respectively. This resulted in the overall performance improvement in terms of f-score values.

We have used the following heuristics to further improve the performance of each of the classifiers. Some of the rules are useful to improve the recall values, whereas

Table 2. Results with context features and second best tag

\begin{tabular}{|c|c|r|r|r|r|r|}
\hline \multicolumn{3}{|l|}{ Baseline + Context features } & \multicolumn{4}{|c|}{ Baseline + Context features + Second best tags } \\
\hline Model & R (in \%) & P (in \%) & FS (in \%) & R (in \%) & P (in \%) & FS (in \%) \\
\hline ME & 79.24 & 72.50 & 75.72 & 85.10 & 74.75 & 79.59 \\
\hline CRF & 82.97 & 77.00 & 79.87 & 88.72 & 76.59 & 82.21 \\
\hline SVM & 83.07 & 77.86 & 80.38 & 90.17 & 77.13 & 83.14 \\
\hline
\end{tabular}


some are effective to increase the precisions. Many of the heuristics are also helpful to identify the boundaries properly. Following are the set of heuristics.

(a). The NNE tag of a particular word is replaced by the appropriate NE tag, if that word appears somewhere in the output with that NE tag.

(b). If any word is tagged as B-XXX/I-XXX/E-XXX (XXX: PER/LOC/ORG/MISC) and the previous and next words are tagged as NNE then that word is assigned the NE tag of type XXX.

(c). The NNE tag of a word is replaced by the E-XXX if the previous word is already tagged as B-XXX.

(d). NNE tag of a word is replaced by B-XXX, if the next word is already tagged as $\mathrm{E}-\mathrm{XXX}$.

(e). If there is sequence $\mathrm{B}-\mathrm{XXX} / \mathrm{I}-\mathrm{XXX}$ followed by $\mathrm{XXX}$ in the output, then the tag $\mathrm{XXX}$ is replaced by the $\mathrm{E}-\mathrm{XXX}$.

(f). If the sequence of tags is of the form XXX B-XXX1/I-XXX1/E-XXX1 NNE (XXX\#XXX1) for three consecutive words in the output, then the tag B-XXX1/I$\mathrm{XXX} 1 / \mathrm{E}-\mathrm{XXX} 1$ is replaced by the XXX1.

(g). If there is a word (current), tagged not as B-XXX/I-XXX/NNE followed by a word that is tagged as $\mathrm{B}-\mathrm{XXX} / \mathrm{I}-\mathrm{XXX} / \mathrm{E}-\mathrm{XXX}$ then the current word is assigned the tag B-XXX.

(h). If the words, tagged as NNE, contain the variable length NE suffixes (used as the feature in the baseline models) then the words are assigned the NE tags. The types of the NE tags are determined by the types of the suffixes (e.g., Person tag is assigned if matches with the person name suffix).

We have presented the experimental results of the classifiers in Table 4 for each heuristics. It shows how each heuristics improve the performance.

Table 3. Results using each heuristics FS (Model): Denotes the f-score for a particular classifier, $\mathrm{H}[\mathrm{i}, 0]$ : Heuristics of the ith row and $0^{\text {th }}$ column of the table

\begin{tabular}{|l|l|l|l|}
\hline Heuristic & FS (ME) & FS (CRF) & FS (SVM) \\
\hline $\mathrm{a}$ & 80.65 & 83.23 & 84.02 \\
\hline $\mathrm{b}+\mathrm{H}[2,0]$ & 80.98 & 83.47 & 84.56 \\
\hline $\mathrm{c}+\mathrm{H}[3,0]$ & 81.34 & 83.79 & 84.83 \\
\hline $\mathrm{d}+\mathrm{H}[4,0]$ & 81.62 & 84.22 & 85.25 \\
\hline $\mathrm{e}+\mathrm{H}[5,0]$ & 82.91 & 84.63 & 85.57 \\
\hline $\mathrm{f}+\mathrm{H}[6,0]$ & 83.12 & 84.89 & 85.82 \\
\hline $\mathrm{g}+\mathrm{H}[7,0]$ & 83.26 & 85.02 & 85.91 \\
\hline $\mathrm{h}+\mathrm{H}[8,0]$ & 83.42 & 85.23 & 86.29 \\
\hline
\end{tabular}

\subsection{Voting}

Voting is a very widely used term and generally used to combine a set of classifiers together into a final system. It is better to give importance to the outputs of all the classifiers rather than giving importance to the output of any particular classifier. It is possible to assign varying weights to the models in order to give more priority to one model than the others. The voting scheme becomes effective in order to improve the 
overall performance of any system. Here, we have combined the three classifiers by simply assigning equal weight to each model and selecting the classifications, which were proposed by the majority of models. The final voted system has demonstrated the recall, precision, and f-score values of $\mathbf{9 0 . 2 3 \%}, \mathbf{8 6 . 0 7 \%}$, and $\mathbf{8 8 . 1 \%}$, respectively. Hence, we observe the improvement the f-score values of $4.68 \%, 2.87 \%$, and $1.81 \%$ in the ME, CRF, and SVM, respectively.

\subsection{Results of the Test Set}

Three systems are tested with a gold standard test set of $40 \mathrm{~K}$ wordforms. Approximately, $21 \%$ of the NEs are unknown in the test set. Experimental results are shown in Table 4. Meaning of the notations used in the experimental results are given below: FS (Bs): F-Score of the baseline model; FS (Bs + C): F-Score using context features; FS (Bs +C+ S): F-Score using context features and second best tag; FS (Bs + $\mathrm{C}+\mathrm{S}+\mathrm{H})$ : F-Score using context features, second best tag and heuristics.

Evaluation results show the f-score values of $73.92 \%, 76.35 \%$, and $77.23 \%$ for the baseline models of ME, CRF, and SVM, respectively. Evaluation results have demonstrated the fact that the use of context features, second best tag and post-processing can improve the performance of each individual system by the impressive margins of 9.5\%, $8.85 \%$, and $9.06 \%$ in f-scores over the baseline ME, CRF, SVM, respectively. These post-processed systems are then combined together into a final system by using majority voting. Experimental results have shown the recall, precision, and f-score values of $\mathbf{9 0 . 7 8 \% , ~} \mathbf{8 7 . 3 5 \%}$, and $\mathbf{8 9 . 0 3 \%}$, respectively. Clearly, this is the improvement in performance compared to any individual system.

Table 4. Results for the test set

\begin{tabular}{|c|c|c|c|c|}
\hline Model & FS (Bs) & FS $(\mathrm{Bs}+\mathrm{C})$ & FS(Bs + C+S $)$ & FS $(\mathrm{Bs}+\mathrm{C}+\mathrm{S}+\mathrm{H})$ \\
\hline ME & 73.92 & 76.11 & 79.65 & 83.42 \\
\hline CRF & 76.35 & 79.22 & 81.17 & 85.23 \\
\hline SVM & 77.23 & 80.13 & 82.23 & 86.29 \\
\hline
\end{tabular}

\subsection{Comparison with Other NER Systems}

The most recent existing Bengali NER systems, i.e., HMM based system [9], CRF based system 10 and SVM based system [11] have been trained and tested with the same datasets. Evaluation results are presented in Table 5. Results show the effectiveness of the proposed NER model that outperforms the other existing Bengali NER systems by an impressive margin. The system outperforms the HMM based system with $\mathbf{1 7 . 2 7 \%}$ f-sore, CRF based system with $\mathbf{9 . 2 2 \%}$ f-score and SVM based system with $\mathbf{9 . 0 7 \%}$ f-score. Thus, it can be decided that purely statistical approaches cannot always perform well across the domain (here, both training and test sets are collected from the mixed domains). So, post-processing the output of the statistical framework with the context patterns, second best tags and several heuristics can yield a reasonably good performance. Results also suggest that combination of several classifiers is more effective than the single classifier. 
Table 5. Comparisons with other models

\begin{tabular}{|l|c|c|c|}
\hline Model & Recall (in \%) & Precision (in \%) & F-Score (in \%) \\
\hline HMM & 73.02 & 70.55 & 71.76 \\
\hline CRF & 80.46 & 79.17 & 79.81 \\
\hline SVM & 81.98 & 78.04 & 79.96 \\
\hline Voted System (proposed) & 90.78 & 87.35 & 89.03 \\
\hline
\end{tabular}

\section{Conclusion}

In this paper, we have developed a NER system for Bengali by combining the ME, CRF and SVM classifiers using a majority voting approach. Performance of each baseline classifier has been improved by the context pattern induction method, second best tag and several heuristics. It has been experimentally shown that post-processing the output of each classifier is effective and improves the performance significantly. The proposed approach shows the effectiveness with the reasonably high recall, precision and f-score values. We have shown that the reported system outperforms the other existing Bengali NER systems. This approach can be applicable for NER in other languages, especially for the Indian languages. Future works include investigating how the proposed approach effect to each of the NE classes. We would like to observe the effect by recognizing miscellaneous expressions using some regular expressions and adopting weighted voting scheme.

\section{References}

1. Bikel, D.M., Schwartz, R., Weischedel, R.M.: An Algorithm that Learns What's in Name. Machine Learning (Special Issue on NLP), 1-20 (1999)

2. Bothwick, A.: A Maximum Entropy Approach to Named Entity Recognition. Ph.D. Thesis, New York University (1999)

3. Lafferty, J., McCallum, A., Pereira, F.: Conditional Random Fields: Probabilistic Models for Segmenting and Labeling Sequence Data. In: Proc. of 18th ICML, pp. 282-289 (2001)

4. Hiroyasu, Y., Kudo, T., Matsumoto, Y.: Japanese Named Entity Extraction using Support Vector Machine. Transactions of IPSJ 43(1), 44-53 (2003)

5. Wu, D., Ngai, G., Carpuat, M.: A Stacked, Voted, Stacked Model for Named Entity Recognition. In: Proceedings of CoNLL 2003 (2003)

6. Florian, R., Ittycheriah, A., Jing, H., Zhang, T.: Named Entity Recognition through Classifier Combination. In: Proceedings of CoNLL 2003 (2003)

7. Munro, R., Ler, D., Patrick, J.: Meta-learning Orthographic and Contextual Models for Language Independent Named Entity Recognition. In: Proceedings of CoNLL 2003 (2003)

8. Ekbal, A., Bandyopadhyay, S.: Lexical Pattern Learning from Corpus Data for Named Entity Recognition. In: Proc. of 5th ICON, India, pp. 123-128 (2007)

9. Ekbal, A., Naskar, S., Bandyopadhyay, S.: Named Entity Recognition and Transliteration in Bengali. Named Entities: Recognition, Classification and Use, Special Issue of Lingvisticae Investigationes Journal 30(1), 95-114 (2007)

10. Ekbal, A., Haque, R., Bandyopadhyay, S.: Named Entity Recognition in Bengali: A Conditional Random Field Approach. In: Proc. of IJCNLP 2008, pp. 589-594 (2008) 
11. Ekbal, A., Bandyopadhyay, S.: Bengali Named Entity Recognition using Support Vector Machine. In: Proc. of NERSSEAL, IJCNLP 2008, pp. 51-58 (2008)

12. Li, Wei, McCallum, Andrew: Rapid Development of Hindi Named Entity Recognition Using Conditional Random Fields and Feature Inductions. ACM TALIP 2(3), 290-294 (2003)

13. Cucerzan, S., Yarowsky, D.: Language Independent Named Entity Recognition Combining Morphological and Contextual Evidence. In: Proc. of the Joint SIGDAT Conference on EMNLP and VLC, pp. 90-99 (1999)

14. Saha, S., Sarkar, S., Mitra, P.: A Hybrid Feature Set based Maximum Entropy Hindi Named Entity Recognition. In: Proc. of IJCNLP 2008, pp. 343-349 (2008)

15. Kumar, N., Bhattacharyya, P.: Named Entity Recognition in Hindi using MEMM. Technical Report, IIT Bombay, India (2006)

16. Ekbal, A., Bandyopadhyay, S.: A Web-based Bengali News Corpus for Named Entity Recognition. Language Resources and Evaluation Journal 40 (2008)

17. Niu, C.g., Li, W., Ding, J., Srihari, R.: A Bootstrapping Approach to Named Entity Classification Using Sucessive Learners. In: Proc. of ACL 2003, pp. 335-342 (2003)

18. Ekbal, A., Haque, R., Bandyopadhyay, S.: Bengali Part of Speech Tagging using Conditional Random Field. In: Proc. of SNLP, Thailand (2007) 